\title{
Multiple Duodenal Diverticula: A Rare Cause of Massive Upper Gastrointestinal Bleeding and Hemodynamic Instability
}

\author{
Nneoma Okoronkwo ${ }^{\mathrm{a}, \mathrm{c}}$, Sami Samiullah ${ }^{\mathrm{b}}$, Gustavo Churrango ${ }^{\mathrm{a}}$, \\ Weizheng Wang ${ }^{\mathrm{b}}$
}

\begin{abstract}
Duodenal diverticula are usually asymptomatic and identified incidentally on imaging studies. In this report, we present a case of massive gastrointestinal (GI) bleeding from multiple duodenal diverticula. A 43-year-old man was evaluated for lightheadedness, hematemesis, and melena. Patient was hypotensive and resuscitated with intravenous fluids and blood products. Upper endoscopy revealed multiple diverticula in the bulb, sweep and second portion of the duodenum with active bleeding from specific diverticulum. Epinephrine was injected around the bleeding diverticulum and multiple endoclips were applied. Patient continued to have persistent melena and required multiple units of packed red blood cells. Repeat endoscopy showed active bleeding from another duodenal diverticulum controlled with endoclips. Patient still had persistent bleeding, thus angiography was performed. Celiac artery stenosis was visualized with poststenotic dilatation. Embolization of the gastroduodenal artery could not be performed due to its retrograde flow supplying the hepatic artery proper. Patient underwent exploratory laparotomy with no further bleeding visualized through a gastrostomy. Duodenum diverticulum is a rare cause of massive upper GI hemorrhage. Endoscopy might not be successful in identifying the bleeding site. There is a high incidence of re-bleeding with endoscopic interventions. Surgery remains the definitive treatment in cases with life-threatening hemorrhage.
\end{abstract}

Keywords: Diverticulum; Gastrointestinal hemorrhage; Upper gastrointestinal tract; Hemostasis

\section{Introduction}

Duodenal diverticula were first described in 1710 by a French

Manuscript accepted for publication July 01, 2015

aDepartment of Medicine, Rutgers New Jersey Medical School, Newark, NJ, USA

bDivision of Gastroenterology and Hepatology, Rutgers New Jersey Medical School, Newark, NJ, USA

${ }^{\mathrm{c} C}$ Corresponding Author: Nneoma Olivia Okoronkwo, Department of Medicine, Rutgers New Jersey Medical School, 150 Bergen Street, UH-I248, Newark, NJ 07103, USA. Email: nneomaokoronkwo1@gmail.com

doi: http://dx.doi.org/10.14740/jmc2229w pathologist Chomel [1]. About $67 \%$ are located in the second portion of the duodenum. The prevalence in the general population ranges from $0.06 \%$ to $22 \%$ depending upon mode of diagnosis. Higher prevalence was reported in autopsy studies [2, 3]. Prevalence ranges from 1:1 to an increased prevalence in men as compared to women [4]. Majority of the cases of duodenal diverticula are asymptomatic and identified incidentally on investigative studies [5]. The most common clinical presentation in patients with symptomatic diverticula is abdominal pain [6]. Complications are rare but can result in significant mortality [2]. Complications of duodenal diverticula include mechanical obstruction of the common bile duct, pancreatic duct or duodenum, diverticulitis with perforation, abscess, fistula formation, sepsis and hemorrhage [2]. Hemorrhage from duodenal diverticula is rare and the first case was reported in 1951 by Patterson and Bromberg $[3,7]$. We present a case of massive gastrointestinal (GI) bleeding from multiple duodenal diverticula.

\section{Case Report}

A 43-year-old man with no significant past medical history was evaluated for dizziness, lightheadedness, hematemesis, and melena. Patient was hypotensive with a systolic blood pressure of $80 \mathrm{~mm} \mathrm{Hg}$ and was resuscitated with intravenous fluids and blood products. An emergent esophagogastroduodenoscopy (EGD) revealed multiple diverticula in bulb, sweep and second portion of the duodenum with active bleeding from diverticulum (Fig. 1, 2). Diluted epinephrine $(1: 10,000)$ was injected around bleeding diverticulum and multiple endoclips were applied to achieve hemostasis (Fig. 3 ). Patient continued to have persistent melena and required multiple units of packed red blood cells, a total of about 20 units throughout his hospital course. A repeat EGD showed active bleeding from another duodenal diverticulum. Endoscopic therapy with epinephrine injection and endoclips was used to achieve hemostasis again. Patient again began to hemorrhage, thus angiography was performed. Angiography showed celiac artery stenosis with poststenotic dilatation. There was retrograde flow of the gastroduodenal artery and was a major arterial supply to hepatic artery proper (Fig. 4). Hence, embolization of gastroduodenal artery was not performed due to risk ischemia to the liver. Patient was thus re- 


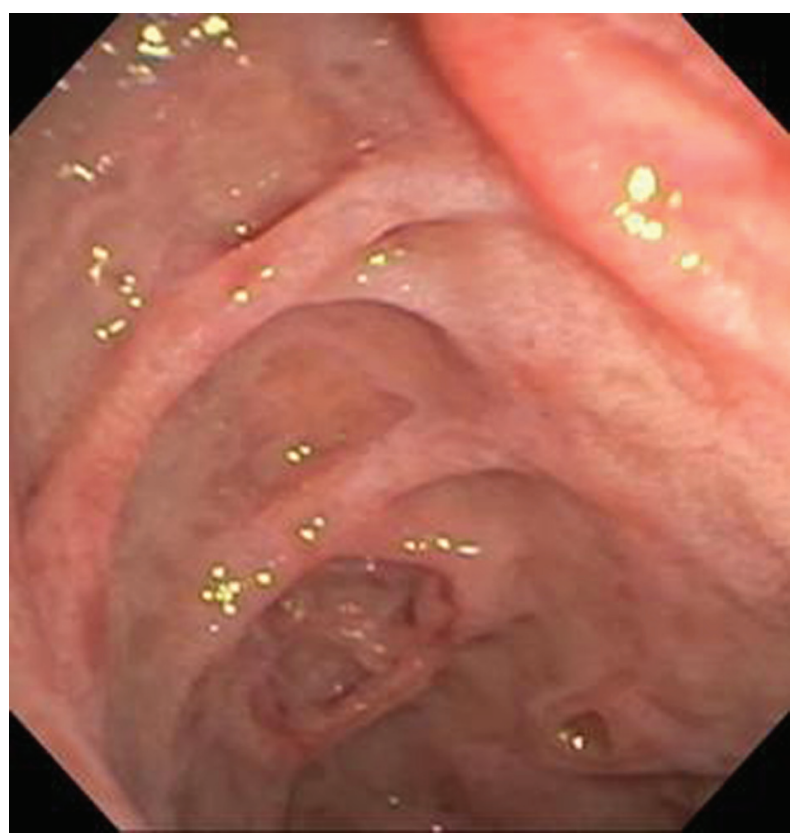

Figure 1. Upper endoscopy showing multiple duodenal diverticula at the duodenal bulb.

ferred for surgical management. Patient underwent exploratory laparotomy. Incidentally, large $4 \mathrm{~cm}$ extra luminal mass was found and excised in its entirety from the anterior wall of the antrum. Through a gastrostomy, no bleeding was visualized from ampulla or diverticulum. The patient did not have any further episodes of bleeding and was discharged home. Pathology of gastric mass was consistent with GI stromal tumor.

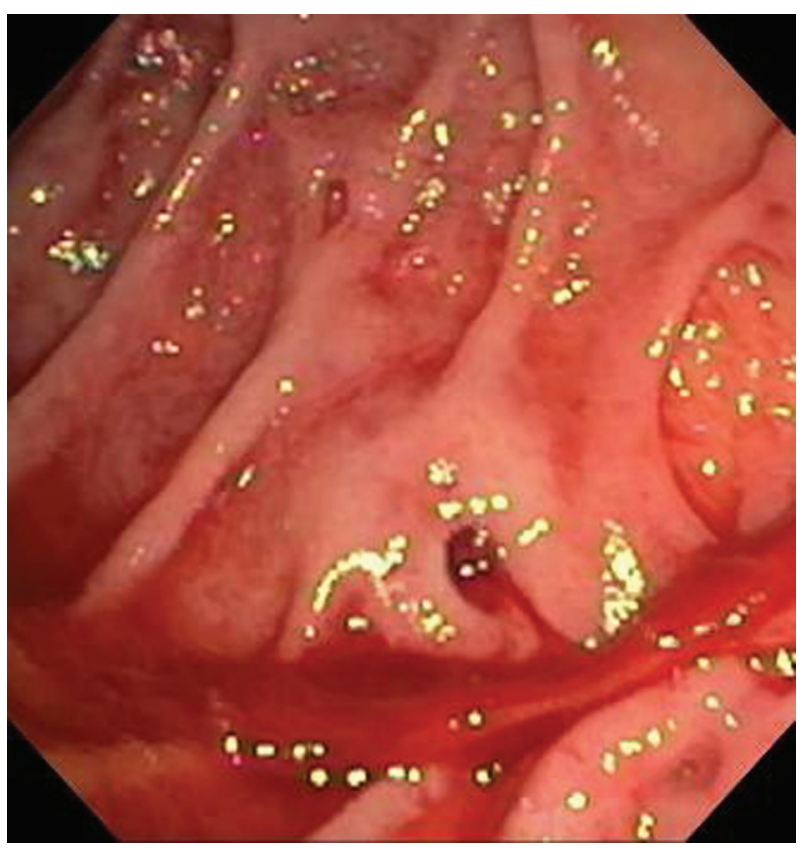

Figure 2. Upper endoscopy showing active hemorrhage and clot formation from a duodenal diverticulum.

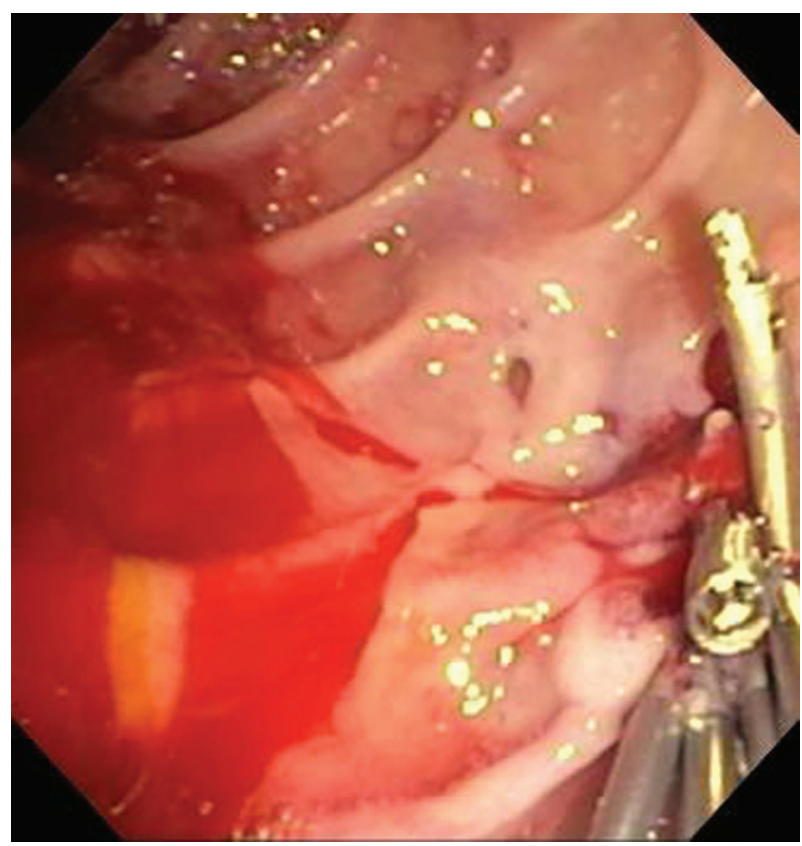

Figure 3. Repeat upper endoscopy showing continued active bleeding from another duodenal diverticulum with six endoclips applied to achieve hemostasis.

\section{Discussion}

Duodenum diverticula are rare cause of massive upper GI hemorrhage. It is estimated that $0.14 \%$ of upper GI bleeding is caused by duodenal diverticula [4]. They are more common in older patients and colonic diverticula may also be present in about $30 \%$ of patients [8]. Most duodenal diverticula are acquired, and consist of a sac of mucosal or sub mucosal layers herniating through a muscular defect or weak point in bowel wall $[3,8]$. However, the exact pathogenesis remains somewhat uncertain $[3,8]$. More than $90 \%$ of duodenal diverticula occur

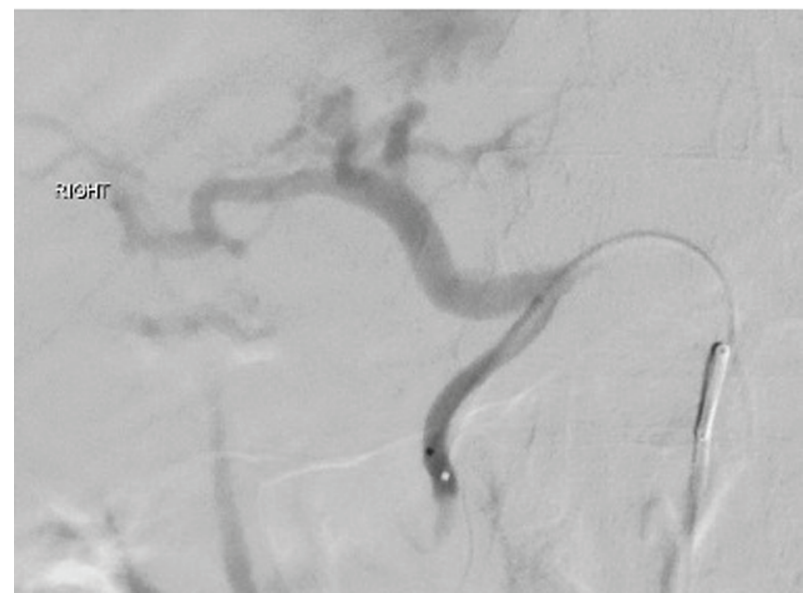

Figure 4. Angiogram showing a 3 French microcatheter used to selectively cannulate the gastroduodenal artery from the celiac axis with retrograde flow noted within the gastroduodenal artery from the superior mesenteric artery. 
on the medial side of the duodenum in second, third and fourth portion. The second portion is the most common site within $2.5 \mathrm{~cm}$ of the major duodenal papilla $[2,3,9,10]$. Hemorrhage has been reported to occur more commonly with diverticula located in the third and fourth portion of the duodenum [8].

The reported incidence of bleeding from duodenal diverticula is about $7 \%$ [10]. The exact mechanism is unknown but some studies have implicated the presence of ectopic gastric mucosa or aortoenteric fistulas, erosion into a major vessel or stasis-induced ulceration as the cause of diverticular bleed $[3,5,8]$. Clinically, significant bleeding from duodenal diverticula usually presents as melena [5]. Presentations as hematemesis and hematochezia have been rarely described [5]. The use of EGD as an initial diagnostic approach offers the advantage of possibly identifying the bleeding site and investigating the presence of other more common etiologies of upper GI bleeding [5]. However, EGD might not be successful in identifying the bleeding site especially if the bleeding is massive or located at a distal site. Other diagnostic modalities such as a computed tomography scan with contrast, magnetic resonance imaging or upper GI barium studies might be helpful in identifying the diverticulum $[4,5]$.

Treatment of duodenal diverticula is generally only recommended in symptomatic cases [4]. Currently, no guidelines exist on the optimal therapeutic approach to manage a bleeding diverticulum. Treatment options include endoscopic techniques to achieve hemostasis, selective arterial embolization and surgical management $[2,3,10]$. The first case of endoscopic hemostasis of duodenal diverticula bleeding was reported in 1991 by Sim et al [7]. However, the use of endoscopy as the sole management can be limited due to the high incidence of re-bleeding [10]. In our case, the patient required endoscopic therapy twice to achieve hemostasis; however, the patient had re-bleeding. Selective embolization was not feasible given the presence of celiac artery stenosis and retrograde flow of gastroduodenal artery. Surgery remains the definitive treatment in cases with persistent life-threatening hemorrhage [10].

In conclusion, duodenal diverticula are a rare cause of upper GI bleeding but should always be considered in patients with an unknown etiology for upper GI bleeding. Cases of successful endoscopic management have been described in literature but there is a significant incidence of re-bleeding. No clear consensus on the therapeutic approach exists but the selection should be on a personalized basis depending on the location, patient's individual risk factors and hemodynamic significance of the hemorrhage.

\section{Grant Support}

None.

\section{Conflict of Interest}

No.

\section{References}

1. Yin WY, Chen HT, Huang SM, Lin HH, Chang TM. Clinical analysis and literature review of massive duodenal diverticular bleeding. World J Surg. 2001;25(7):848-855.

2. Rioux L, Des Groseilliers S, Fortin M, Mutch DO. Massive upper gastrointestinal bleeding originating from a fourth-stage duodenal diverticulum: a case report and review of the literature. Can J Surg. 1996;39(6):510-512.

3. Onozato Y, Kakizaki S, Ishihara H, Iizuka H, Sohara N, Okamura S, Mori M, et al. Endoscopic management of duodenal diverticular bleeding. Gastrointest Endosc. 2007;66(5):1042-1049.

4. Wilhelmsen M, Andersen JF, Lauritsen ML. Severe upper gastrointestinal bleeding in extraluminal diverticula in the third part of the duodenum. BMJ Case Rep. 2014;2014.

5. Oukachbi N, Brouzes S. Management of complicated duodenal diverticula. J Visc Surg. 2013;150(3):173-179.

6. Kouraklis G, Glinavou A, Mantas D, Kouskos E, Karatzas G. Clinical implications of small bowel diverticula. Isr Med Assoc J. 2002;4(6):431-433.

7. Dan D, Bascombe N, Maharaj R, Hariharan S, Naraynsingh V. Laparoscopic diverticulectomy for massive hemorrhage in a duodenal diverticulum. Surg Laparosc Endosc Percutan Tech. 2012;22(1):e39-41.

8. Dalal AA, Rogers SJ, Cello JP. Endoscopic management of hemorrhage from a duodenal diverticulum. Gastrointest Endosc. 1998;48(4):418-420.

9. Wong LS, Hobbs S, Dorricott NJ. Duodenal diverticulum as a cause of massive gastrointestinal bleeding. J R Soc Med. 1999;92(5):254-255.

10. Seneviratne SA, Samarasekera DN. Massive gastrointestinal haemorrhage from a duodenal diverticulum: a case report. Cases J. 2009;2:6710. 